\title{
Associations of serum indolepropionic acid, a gut microbiota metabolite, with type 2 diabetes and low-grade inflammation in high-risk individuals
}

\author{
Marjo Tuomainen', Jaana Lindström², Marko Lehtonen ${ }^{3,4}$, Seppo Auriolal, ${ }^{3,4}$ Jussi Pihlajamäki ${ }^{1,5}$, Markku Peltonen²,

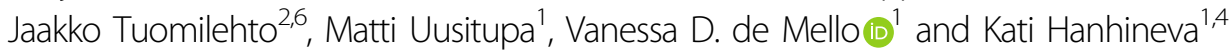

\begin{abstract}
We recently reported using non-targeted metabolic profiling that serum indolepropionic acid (IPA), a microbial metabolite of tryptophan, was associated with a lower likelihood of developing type 2 diabetes (T2D). In the present study, we established a targeted quantitative method using liquid chromatography with mass spectrometric detection (HPLC-QQQ-MS/MS) and measured the serum concentrations of IPA in all the participants from the Finnish Diabetes Prevention Study (DPS), who had fasting serum samples available from the 1-year study follow-up $(n=209$ lifestyle intervention and $n=206$ control group). Higher IPA at 1-year study was inversely associated with the incidence of T2D (OR [Cl]: $0.86[0.73-0.99], P=0.04)$ and tended to be directly associated with insulin secretion $(\beta=0.10, P=0.06)$ during the mean 7-year follow-up. Moreover, IPA correlated positively with dietary fiber intake ( $\mathrm{g} /$ day: $r=0.24$, $\left.P=1 \times 10^{-6}\right)$ and negatively with hsCRP concentrations at both sampling $(r=-0.22, P=0.0001)$ and study follow-up $(\beta=-0.19, P=0.001)$. Thus, we suggest that the putative effect of IPA on lowering T2D risk might be mediated by the interplay between dietary fiber intake and inflammation or by direct effect of IPA on $\beta$-cell function.
\end{abstract}

\section{Introduction}

Well-established lifestyle, and metabolic and genetic factors are currently used for stratifying people at high risk of developing type 2 diabetes (T2D). Even though physical inactivity, overweight, and obesity are generally accepted major risk factors contributing to the T2D incidence $^{1}$, the quality of the diet seems also to have a role.

We recently performed a non-targeted metabolite profiling study in pre-selected participants with impaired

\footnotetext{
Correspondence: Vanessa D. de Mello (Vanessa.Laaksonen@uef.fi) or Kati Hanhineva (Kati.Hanhineva@uef.fi)

${ }^{1}$ Department of Clinical Nutrition, Institute of Public Health and Clinical

Nutrition, University of Eastern Finland, Kuopio, Finland

${ }^{2}$ Department of Chronic Disease Prevention, National Institute for Health and Welfare, Helsinki, Finland

Full list of author information is available at the end of the article.

These authors share last authorship: Vanessa D. de Mello and Kati Hanhineva.
}

glucose tolerance (IGT) from the Finnish Diabetes Prevention Study (DPS) and reported that serum indolepropionic acid (IPA) was associated with a lower likelihood of developing $\mathrm{T}^{2} \mathrm{D}^{2}$. Furthermore, we replicated this association in two other independent cohorts ${ }^{2}$. In DPS, IPA was the only metabolite linked with preservation of $\beta$-cell function in those who did not develop T2 $\mathrm{D}^{2}$.

IPA is a specific microbial product from dietary tryptophan absorbed from the gut into the blood stream and is also found in cerebrospinal fluid ${ }^{3,4}$. In animal models, IPA exerts antioxidant and anti-inflammatory effects and possibly ameliorates glucose metabolism ${ }^{5,6}$.

Because of the putative link of gut microbiota and $\mathrm{T}_{2} \mathrm{D}^{7}$, we aimed at getting a more accurate picture of the interplay between IPA, T2D, glucose metabolism, inflammation, and diet. Therefore, we established a targeted quantitative method using liquid chromatography 
with triple quadrupole mass spectrometric detection (HPLC-QQQ-MS/MS) to measure the precise concentrations of IPA in serum samples from the DPS study.

\section{Research design and methods Study participants}

The DPS was a randomized, controlled, multicenter study carried out in Finland between the years 1993 and 2001 (ClinicalTrials.gov NCT00518167). A total of 522 individuals with IGT were randomly allocated into either a lifestyle intervention or control group. After a mean 4year intervention (active study) period, the postintervention follow-up was carried out with annual examinations. The DPS study design and methods have been reported in detail elsewhere ${ }^{8}$ and are described in the Supplementary Information (SI) material.

The present study included all the participants who had fasting serum samples available for IPA analysis from the one-year follow-up. Altogether, IPA was measured in serum of 415 participants $(n=209$ lifestyle and $n=206$ control groups, respectively).

\section{Laboratory determinations and genotyping}

Glucose and insulin levels were determined as previously described $^{9}$ and as surrogate index of the first/early-phase insulin secretion we used the disposition index ${ }_{30}\left(\mathrm{DI}_{30}\right)^{9}$ (details in SI material). High sensitive C-reactive protein (hSCRP) was measured in fasting serum at IPA sampling (1-year follow-up) and yearly during the mean 4-year intervention (active study) period using an IMMULITE $^{\circledR}$ 2000 Systems Analyzer (Siemens Healthcare Diagnostics, Inc. Tarrytown, NY) ${ }^{2}$. Genotyping of TCF7L2 rs7903146 and rs12255372 was performed as reported ${ }^{10}$.

\section{Quantitation with HPLC-MS/MS}

IPA was quantified by HPLC-QQQ-MS/MS using reversed-phase separation technique. Commercial IPA and IPA-d2 were used as a standard and internal standard, respectively. Details on materials, sample preparation, HPLC-QQQ-MS/MS system, and method validation are described in the SI material.

\section{Statistical analyses}

The data were analyzed using IBM SPSS Statistics 23 software (IBM, Inc., Armonk, NY). After data normalization, Cox proportional hazards regression models assessed the association of IPA with the risk of incident T2D during a mean follow-up of 7 years (range 1-14 years) after IPA sampling. In addition, analysis of variance models adjusted for study group tested the associations of IPA with TCF7L2 genotypes and with insulin secretion $\left(\mathrm{DI}_{30}\right)$ during the long-term follow-up. For testing correlations, we applied Pearson's correlation test. A value of $P<0.05$ was considered significant.

\section{Results}

\section{IPA concentration and diabetes incidence}

After quality control (detailed in SI material), 403 samples (202 lifestyle intervention and 201 control) were included in the final analyses (Table 1). IPA concentration was not different between the study groups $(P=0.14$, Table 1). When participants with incident T2D at IPA sampling (1-year examination) were excluded $(n=4$, intervention and $n=13$, control), the results remained the same $(P=0.12)$.

During a mean follow-up of 7 years since IPA sampling, the number of diabetes cases was 95 in the control and 71 in the intervention groups. Participants who progressed from IGT to diabetes compared with those who did not had reduced levels of IPA at 1-year follow-up (169 [104-264] vs. 207 [118-304] ng/ml, respectively; $P=0.05$ ).

We observed that higher IPA concentrations were inversely associated with the incidence of diabetes during the mean 7-year follow-up (odds ratio [confidence interval]: 0.86 [0.73-0.99], $P=0.04)$. A 1 SD increase in IPA was associated with a $14 \%$ decrease in the risk of developing diabetes. However, the association lost its significance when body mass index (BMI) $(P=0.15)$, fasting $(P=0.07)$, or $2 \mathrm{~h}(P=0.26)$ plasma glucose at IPA sampling were also taken into account. In a model where DPS study group, age and sex were included the association of IPA with diabetes incidence was boderline $(P=0.09)$.

\section{IPA and insulin secretion}

The IPA concentrations tended to be directly associated with insulin secretion $\left(\mathrm{DI}_{30}\right)$ during the mean 7 -

Table 1 Characteristics of the participants at serum IPA sampling (1-year examination study) $(n=403)$

\begin{tabular}{llll}
\hline & Intervention (202) & Control (201) & $\boldsymbol{P}^{*}$ \\
\hline Age (years) & $56.2 \pm 7.0$ & $55.0 \pm 7.0$ & 0.11 \\
Sex (male/female) & $70 / 132$ & $58 / 143$ & 0.24 \\
Body weight (kg) & $82.0 \pm 13.4$ & $84.7 \pm 14.5$ & 0.05 \\
BMI (kg/m²) & $29.6 \pm 4.3$ & $30.8 \pm 4.6$ & 0.006 \\
Plasma glucose (mmol/l) & & 0.0001 \\
$\quad$ Fasting & $5.9 \pm 0.7$ & $6.2 \pm 0.9$ & 0.01 \\
120 min & $8.1 \pm 1.9$ & $8.5 \pm 2.1$ & \\
Serum insulin (pmol/l) & $76(63 ; 104)(196)$ & $90(63 ; 118)(193)$ & 0.02 \\
Fasting & $358(236 ; 606)(190)$ & $438(313 ; 705)(189)$ & 0.02 \\
120 min & $192(118 ; 291)$ & $174(104 ; 276)$ & 0.14 \\
\hline IPA (ng/ml)
\end{tabular}

ANOVA analysis of variance, BMI body mass index, IPA indolepropionic acid Data are mean $\pm S D$, median (interquartile range) or $(n)$. ${ }^{*} P$ for the difference between groups at 1-year study using one-way ANOVA for continuous variables or Fisher's exact test for sex variables. 
year follow-up $(\beta=0.10, P=0.06$; Fig. 1a). Models adjusted for sex, age, and DPS group retrieved similar results $\left(P=0.07\right.$ for the effect of IPA on $\mathrm{DI}_{30}$ during the follow-up).

\section{IPA and TCF7L2}

Because of the strong relationship of TCF7L2 genotype with T2D and insulin secretion ${ }^{10,11}$, we tested whether specific related genotypes could interfere in the relationship of IPA with insulin secretion. Overall, these genotypes did not influence IPA concentrations (STable 1) or its association with $\mathrm{DI}_{30}(P>0.30$ for each variant at each respective model).

\section{IPA correlates with dietary fiber and low-grade inflammation}

Our previous results suggested a correlation between IPA and dietary fiber ${ }^{2}$, which was confirmed in the current study (Fig. 1b. $r=0.24, P=1 \times 10^{-6}$ ). There was only a mild correlation between saturated fat intake and IPA, which was no longer significant after controlling for fiber intake (STable 2).

We found a negative correlation of IPA and serum hsCRP levels $(r=-0.22, P=0.0001)$, even after controlling for study group $(P=0.0002)$ or BMI $(P=0.001)$. One-year serum hsCRP was inversely associated with $\mathrm{DI}_{30}$ during the long-term follow-up independently of study group $(\beta=-0.14, P=0.01)$, but not after controlling for BMI $(P=0.30)$.

Serum hsCRP also correlated negatively with fiber intake $\left(r=-0.22, P=6.6 \times 10^{-5}\right)$ after controlling for study group $\left(P=6.6 \times 10^{-5}\right)$ or BMI $(P=0.001)$. When controlled for fiber intake, the correlation between serum IPA and hsCRP concentrations remained significant $(P=$ 0.003). We also observed an impact of serum IPA at 1year study on the average of circulating levels of hsCRP during the 4 years of the study $(\beta=-0.19$, $P=0.001$ ), independently of the study group (Fig. 1c).

\section{Discussion}

We established the quantitative HPLC-QQQ-MS/MS method for measuring serum IPA in the Finnish DPS. We showed a relationship of IPA with the incidence of T2D using the original design of the DPS and demonstrated a trend for an association with insulin secretion during a longer follow-up time of 7 years. Moreover, we demonstrated that higher serum IPA was associated with lower low-grade inflammation and higher dietary fiber intake. In our study, the predictive value of IPA on T2D incidence weakened after controlling for confounding factors. This suggests that healthy lifestyle changes resulting in higher fiber intake that protected from T2D may modify IPA concentrations and consequently diminish any associations in the whole cohort.
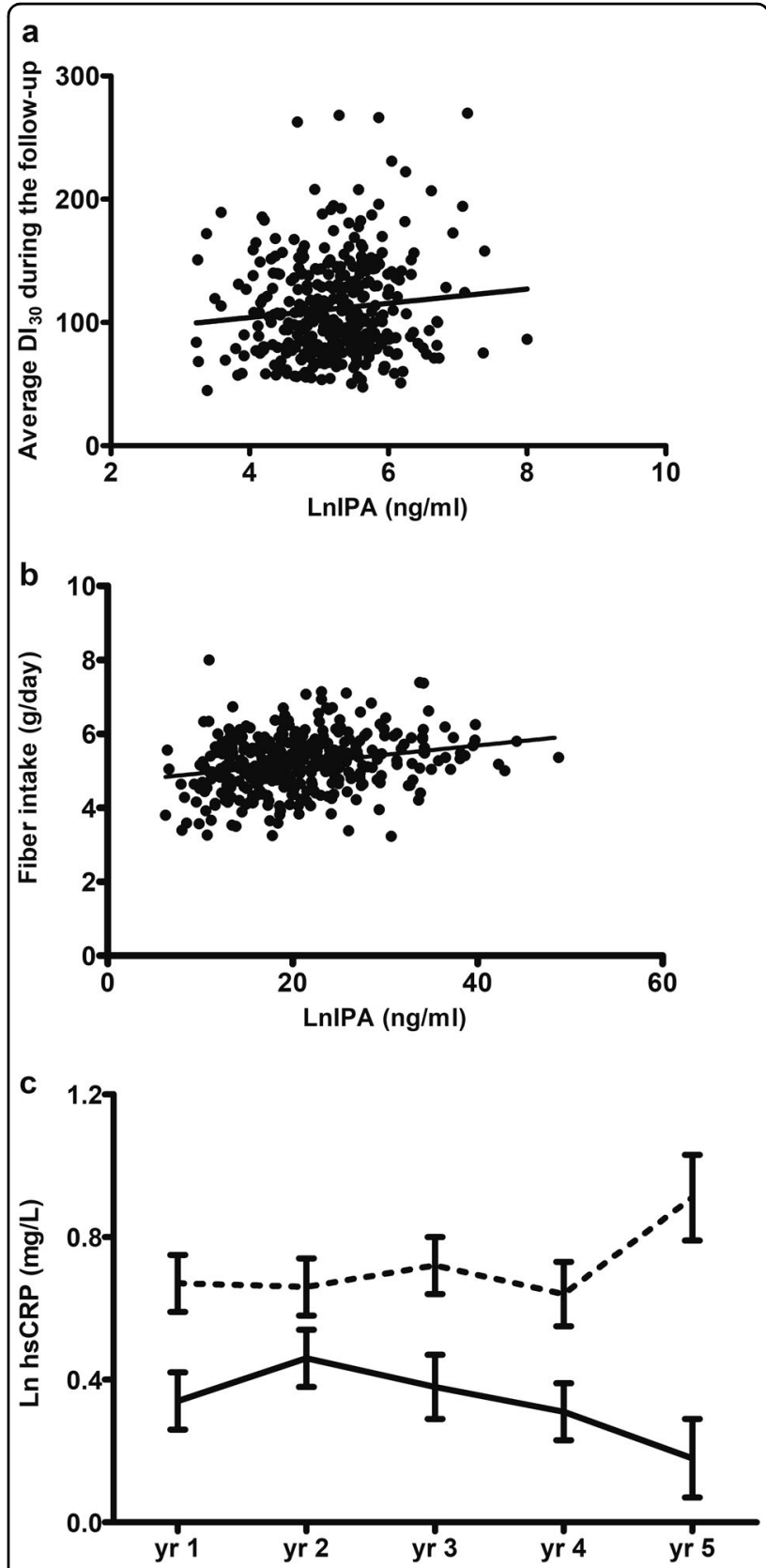

Fig. 1 Scattered plot of the relationship of IPA concentrations (natural log transformed; $\mathrm{ng} / \mathrm{ml}$ ) measured from 1-year examination samples and $\mathbf{a}$. average insulin secretion $\left(\mathrm{Dl}_{30}\right)$ during the mean seven years of follow-up in the DPS $(\beta=0.10, P=0.06)$ and $\mathbf{b}$. fiber intake ( $\mathrm{g} / \mathrm{day})$ at IPA sampling $\left(r=0.24, P=1 \times 10^{-6}\right)$. c. Association of indolepropionic acid (IPA) and hsCRP serum concentrations in DPS $(n=291)$.

Descriptive figure of the course of serum hsCRP (natural log transformed; $\mathrm{mg} / \mathrm{L}$ ) during the active study follow-up since IPA sampling (yr 1) according to the median cut-off point in IPA. Solid line = above median cut-off; broken lines = below median cut-off. $P=0.008$ for the difference between cut-off point groups 
IPA levels correlated negatively with hsCRP concentrations, which has been previously linked with an increased risk of T2D in the DPS population ${ }^{12}$. The gut microbiota seem to have a role in $\mathrm{T}_{2} \mathrm{D}^{7}$. Therefore, the beneficial effect of increasing dietary fiber and concomitant weight loss on gut microbiota could be linked to the production of IPA, which by enhancing intestinal barrier integrity and lowering inflammation ${ }^{13-15}$, ultimately leads to improved insulin secretion as seen in our study, thereby lowering the risk of T2D. In addition, due to IPA modulation of incretin hormones ${ }^{5}$ this could lead to enhanced insulin secretion.

It has been suggested that higher IPA could ameliorate inflammation ${ }^{16}$ and cell oxidative damage ${ }^{17,18}$, thereby resulting on better insulin secretion due to preservation of $\beta$-cells, and consequently lowering the risk of T2D. Accordingly, lower hsCRP concentrations at IPA sampling was associated with a better insulin secretion during the follow-up years. However, it is known that weight loss has an impact on ameliorating beta-cell function and lowering inflammation ${ }^{9,19,20}$. Therefore, it is not surprising that in our study obesity modified the association of insulin secretion with both IPA and inflammation.

Importantly, neither the strongest common T2D-

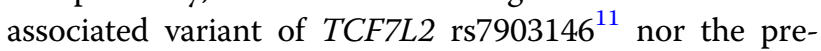
viously reported T2D-associated variant rs7903146 in DPS $^{10}$ modified the association of IPA levels and insulin secretion, confirming that the effect of TCF7L2 is probably not mediated by IPA.

Strengths of the present study include the wellcharacterized and homogenous study population and yearly measurements of insulin secretion estimates during a long follow-up. Moreover, we developed a method for quantification of IPA in serum using all samples available from the DPS. Our study has limitations. The surrogate for insulin secretion $\left(\mathrm{DI}_{30}\right)$ was based on indexes that were not measured by either the hyperinsulinemiceuglycemic clamp or the intravenous glucose tolerance test (IVGTT). Instead, we used an IVGTT for validation?

In conclusion, we propose that the putative beneficial effects of IPA on lowering T2D risk relate to the interplay between high dietary fiber intake and decreased inflammation, or by the direct effect of IPA on $\beta$-cell function. Overall, our study further highlights the importance of the gut microbiota as a mediator for the development of metabolic disorders like T2D.

\section{Acknowledgements}

This study was supported by the Academy of Finland (128315, 129330, and 283454), the Finnish Diabetes Research Foundation, and the Biocenter Finland.

\section{Author details}

${ }^{1}$ Department of Clinical Nutrition, Institute of Public Health and Clinical Nutrition, University of Eastern Finland, Kuopio, Finland. ${ }^{2}$ Department of Chronic Disease Prevention, National Institute for Health and Welfare, Helsinki, Finland. ${ }^{3}$ School of Pharmacy, University of Eastern Finland, Kuopio, Finland.
${ }^{4}$ LC-MS Metabolomics Center, Biocenter Kuopio, Kuopio, Finland. ${ }^{5}$ Clinical Nutrition and Obesity Center, Kuopio University Hospital, Kuopio, Finland. ${ }^{6}$ Dasman Diabetes Institute, Dasman, Kuwait

\section{Author contributions}

J.L., J.T., and M.U. contributed to the study design, acquisition of data, and funding. M.T., V.D.d.M., and M.P. did the statistical analyses. M.T., M.P., J.P., and V.D.d.M. participated in the interpretation of the data. M.T. and V.D.d.M. drafted the article. K.H., S.A., and M.L. have contributed on supervising the data analysis. All the authors have critically contributed to the manuscript and approved the final version. V.D.d.M. and J.L. are the guarantors of this work and, as such, had full access to all the data in the study, and takes responsibility for the integrity of the data and the accuracy of the data analysis.

\section{Conflict of interest}

The authors declare that they have no conflict of interest.

Supplementary Information accompanies this paper at (https://doi.org/ 10.1038/s41387-018-0046-9).

Received: 27 February 2018 Revised: 19 April 2018 Accepted: 1 May 2018 Published online: 25 May 2018

\section{References}

1. Global report on diabetes. 2016.

2. de Mello, V. D. et al. Indolepropionic acid and novel lipid metabolites are associated with a lower risk of type 2 diabetes in the Finnish Diabetes Prevention Study. Sci. Rep. 7, 46337 (2017).

3. Wikoff, W. R. et al. Metabolomics analysis reveals large effects of gut microflora on mammalian blood metabolites. Proc. Natl Acad. Sci. USA 106, 3698-3703 (2009).

4. Young, S. N., Anderson, G. M., Gauthier, S. \& Purdy, W. C. The origin of indoleacetic acid and indolepropionic acid in rat and human cerebrospinal fluid. J. Neurochem. 34, 1087-1092 (1980).

5. Chimerel, $\mathrm{C}$. et al. Bacterial metabolite indole modulates incretin secretion from intestinal enteroendocrine L cells. Cell Rep. 9, 1202-1208 (2014).

6. Zhang, L. S. \& Davies, S.S. Microbial metabolism of dietary components to bioactive metabolites: opportunities for new therapeutic interventions. Genome Med. 8, 46 (2016)

7. Li, X., Watanabe, K. \& Kimura, I. Gut microbiota dysbiosis drives and implies novel therapeutic strategies for diabetes mellitus and related metabolic diseases. Front. Immunol. 8, 1882 (2017).

8. Lindström, J. et al. Improved lifestyle and decreased diabetes risk over 13 years: long-term follow-up of the randomised Finnish Diabetes Prevention Study (DPS). Diabetologia 56, 284-293 (2013).

9. de Mello, V. D. et al. Insulin secretion and its determinants in the progression of impaired glucose tolerance to type 2 diabetes in impaired glucose-tolerant individuals: the Finnish Diabetes Prevention Study. Diabetes Care 35, 211-217 (2012).

10. Wang, J. et al. Variants of transcription factor 7-like 2 (TCF7L2) gene predict conversion to type 2 diabetes in the Finnish Diabetes Prevention Study and are associated with impaired glucose regulation and impaired insulin secretion. Diabetologia 50, 1192-1200 (2007).

11. Morris, A. P. et al. Large-scale association analysis provides insights into the genetic architecture and pathophysiology of type 2 diabetes. Nat. Genet. 44, 981-990 (2012).

12. Herder, C. et al. Systemic immune mediators and lifestyle changes in the prevention of type 2 diabetes: results from the Finnish Diabetes Prevention Study. Diabetes 55, 2340-2346 (2006).

13. Xiao, S. et al. A gut microbiota-targeted dietary intervention for amelioration of chronic inflammation underlying metabolic syndrome. FEMS Microbiol. Ecol. 87, 357-367 (2014).

14. Xiao, S. \& Zhao, L. Gut microbiota-based translational biomarkers to prevent metabolic syndrome via nutritional modulation. FEMS Microbiol. Ecol. 87 303-314 (2014).

15. Jennis M., et al. Microbiota-derived tryptophan indoles increase after gastric bypass surgery and reduce intestinal permeability in vitro and in vivo. Neurogastroenterol. Motil. $\mathbf{3 0}$ (2018) https://doi.org/10.1111/nmo.13178. 
16. Venkatesh, M. et al. Symbiotic bacterial metabolites regulate gastrointestinal barrier function via the xenobiotic sensor PXR and Toll-like receptor 4. Immunity 41, 296-310 (2014).

17. Karbownik, M., Garcia, J. J., Lewinski, A., \& Reiter, R. J. Carcinogen-induced, free radical-mediated reduction in microsomal membrane fluidity: reversal by indole-3-propionic acid. J. Bioenerg. Biomembr. 33, 73-78 (2011).

18. Karbownik, M. et al. Indole-3-propionic acid, a melatonin-related molecule, protects hepatic microsomal membranes from iron-induced oxidative damage: relevance to cancer reduction. J. Cell. Biochem. 81 507-513 (2001).

19. Camastra, S. et al. Muscle and adipose tissue morphology, insulin sensitivity and beta-cell function in diabetic and nondiabetic obese patients: effects of bariatric surgery. Sci. Rep. 7, 9007-017-08444-6 (2017).

20. Eguchi, K. \& Nagai, R. Islet inflammation in type 2 diabetes and physiology. J. Clin. Invest. 127, 14-23 (2017). 